\title{
Near-Lossless Binarization of Word Embeddings
}

\author{
Julien Tissier, Christophe Gravier, Amaury Habrard \\ Univ. Lyon, UJM Saint-Etienne \\ CNRS, Lab Hubert Curien UMR 5516 \\ 42023, Saint-Etienne, France \\ \{julien.tissier, christophe.gravier, amaury.habrard\}@univ-st-etienne.fr
}

\begin{abstract}
Word embeddings are commonly used as a starting point in many NLP models to achieve state-of-the-art performances. However, with a large vocabulary and many dimensions, these floating-point representations are expensive both in terms of memory and calculations which makes them unsuitable for use on low-resource devices. The method proposed in this paper transforms real-valued embeddings into binary embeddings while preserving semantic information, requiring only 128 or 256 bits for each vector. This leads to a small memory footprint and fast vector operations. The model is based on an autoencoder architecture, which also allows to reconstruct original vectors from the binary ones. Experimental results on semantic similarity, text classification and sentiment analysis tasks show that the binarization of word embeddings only leads to a loss of $\sim 2 \%$ in accuracy while vector size is reduced by $97 \%$. Furthermore, a top-k benchmark demonstrates that using these binary vectors is 30 times faster than using real-valued vectors.
\end{abstract}

\section{Introduction}

Word embeddings models play a central role in many NLP applications like document classification (Joulin et al. 2017; Conneau et al. 2017) or sentiment analysis (Socher et al. 2013; Qian et al. 2017). The real-valued vector representation associated to each word of a vocabulary $\mathcal{V}$ reflects its semantic and syntactic information extracted from the language (Bengio et al. 2003). They are usually created from a large corpus by moving closer the vectors of words co-occurring together (Mikolov et al. 2013) or factorizing the matrix containing co-occurrences statistics (Pennington, Socher, and Manning 2014), and commonly require several gigabytes of memory space. For example, with a vocabulary of 2 million words and 300-dimensional vectors, storing the word embedding require 2.4 gigabytes (with real values encoded with 32-bit float).

Motivation Storing and running such models on embedded devices like cell phones is not feasible in practice, due to their limited memory and low computing power for floatingpoint arithmetic. In general, NLP applications running on

Copyright (c) 2019, Association for the Advancement of Artificial Intelligence (www.aaai.org). All rights reserved. smartphones send data to large computing servers that perform the calculations and return the results. The method described in this paper aims to reduce the size of the word embeddings models so that the computations can be done locally on the embedded device. The benefits are twofold:

1. the embedded device can run NLP applications offline;

2. privacy is preserved since no user data is send to servers.

A solution to reduce the size is to binarize the model; either the learning parameters (Hubara et al. 2016) or the vector representations (Joulin et al. 2017). This paper stands in the second category. Associating binary codes to words allows one to speed up calculations as the vector operations can be done with bitwise operators instead of floating-point arithmetic, e.g. computing the distance between two binary vectors only requires a XOR and a popcount ( ) ${ }^{1}$ operations (which are both performed with a single CPU cycle due to hardware optimizations in modern processors) while it requires $\mathcal{O}(n)$ floating-point multiplications and additions for $n$-dimensional real-valued vectors. To take advantage of fast CPU optimized bitwise operations, the size of binary vectors has to be in adequacy with register sizes (64, 128 or 256 bits). When this criteria is met, the computations are much faster (Norouzi, Punjani, and Fleet 2012; Subercaze, Gravier, and Laforest 2015). Nevertheless, mapping words to binary codes is not enough as the vectors are then used in NLP applications. They also need to encode semantic and syntactic information; the objective then becomes to find binary vectors that preserve the language aspects and are small enough to fit in CPU registers.

Contributions This paper solves the problem of producing binary word vectors from real-valued embeddings while preserving semantic information. Our model is based on an autoencoder architecture which transforms any real-valued vectors to binary vectors of any size (more specifically 64, 128 or 256 bits) and allows one to reconstruct original vectors from the binary ones. Our binary vectors use $\mathbf{3 7 . 5}$ times less memory and perform a top-k query 30 times faster than real-valued vectors, with only an accuracy loss of $\sim 2 \%$ on several NLP tasks. Entire source code to generate and evaluate binary vectors is available online ${ }^{2}$.

\footnotetext{
${ }^{1}$ popcount $(n)$ returns the number of bits set to 1 in $n$.

${ }^{2}$ https://github.com/tca19/near-lossless-binarization
} 


\section{Related work}

Dimensionality reduction Classic dimensionality reduction techniques (Raunak 2017) can be used to produce smaller embeddings. Although the number of dimensions can be halved, the produced vectors still require floatingpoint operations when they are used. Another method (Ling, Song, and Roth 2016) limits the precision of each dimension to 4 or 8 bits, but the size of the vectors is not aligned with CPU register sizes. Other approaches (Shu and Nakayama 2018; Chen, Min, and Sun 2018) only store a small set of basis vectors which are then used as linear combinations to produce a vector for each word. The memory size is largely reduced $(\sim 98 \%)$ because they do not store the whole embeddings matrix, but the vectors are still real-valued and do not benefit from the aforementionned calculation gains.

Binary word vectors A naive approach to produce binary word embeddings is to map each value $x$ of a pre-trained real-valued embedding to 0 if $x<0$ and 1 otherwise. This method does not require any training step but suffers from an important drawback: the binary vectors have the same dimension as the original ones. To produce vectors of 64 , 128 or 256 bits, one has to retrain real-valued embedding with these dimensions, and then apply the naive binarization. The results of subsection 5.4 show that it is slower and less efficient than the proposed method that directly transforms vectors with the appropriate binary size.

Semantic hashing is one way to find compact binary codes in order to well approximate nearest neighbor search in the original space. NASH (Shen et al. 2018) finds a binary code to each document for fast information retrieval, using an end-to-end neural architecture. Locality sensitive hashing (Charikar 2002) uses random projections to produce binary codes that approximates the cosine similarity of the corresponding orginal vectors. However, these methods generally fail to fully preserve semantic similarities (Xu et al. 2015).

Faruqui et al. (2015) propose to binarize real-valued vectors by first increasing the vector size to create sparse vectors, and then applying the naive binarization function. Although this method preserves the semantic similarities, the produced vectors are not really small and does not fit in CPU registers. Fasttext.zip (Joulin et al. 2016) binarizes word vectors by clustering them and concatenating the binary codes of the $k$ closest centroids for each word. The binary vectors are then used in a document classification task but this method does not produce generic binary vectors that could be used in other tasks as the vocabulary size is drastically reduced to around 1000 words.

Although binary vectors can speed up vector computations, some NLP applications only work with real-valued vectors (Ma and Hovy 2016); being able to reconstruct realvalued vectors from the binary ones is therefore required for such applications. As a consequence, one can store the binary embeddings and compute on the fly real-valued vectors only for the words it needs instead of storing the real-valued vectors of all words, reducing the required memory space (e.g. for $1 \mathrm{M}$ words and 128-bit vectors, storing binary codes and the reconstruction matrix requires $16.1 \mathrm{MB}$ vs. $1.2 \mathrm{~GB}$ for real-valued vectors).

\section{Binarizing word vectors with autoencoder}

\subsection{Autoencoder architecture}

Let $\mathcal{V}$ be a vocabulary of words, and $X \in \mathbb{R}^{|\mathcal{V}| \times m}$ a $(|\mathcal{V}| \times m)$ matrix whose rows are $m$-dimensional real-valued vectors representing the embedding of each word. Our main objective is to transform each row $x_{i}$ into a binary representation $b_{i}$ of dimension $n$ with $n \ll k \times m$ where $k$ corresponds to the encoding size of real-valued numbers (e.g. 32 bits for standard single-precision floating-point format) while preserving the semantic information contained in $x_{i}$. The proposed method achieves this objective by using an autoencoder model composed of two parts: an encoder that binarizes the word vector $x$ to $\Phi(x)$ and a decoder that reconstructs a real-valued vector from $\Phi(x)$. The binarized vector is therefore the latent representation of the autoencoder. Figure 1 summarizes this architecture.

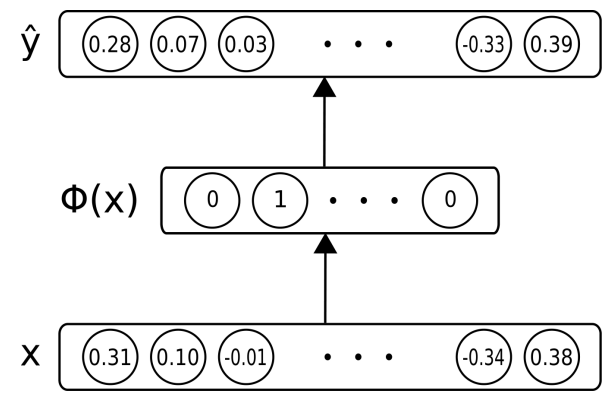

Figure 1: Autoencoder architecture used to binarize word vectors and reconstruct a real-valued representation. The model can learn any vector size for $\Phi(x)$.

Encoding to binary vectors Let $W$ be a $n \times m$ matrix. We define the binarized vector $b_{i}$ of $x_{i}$ as:

$$
b_{i}=\Phi\left(x_{i}\right)=h\left(W \cdot x_{i}^{T}\right)
$$

where $h($.$) is an element-wise function that outputs a bit$ given a real value such as the Heaviside step function. The dimension of $W$ is $(n \times m)$ i.e. the desired binary size and the size of the original real-valued vector. Therefore our model can be used to generate binary vectors of any size independently of the original size of the real-valued vector.

Reconstructing a real-valued vector The generated representation $\Phi\left(x_{i}\right)$ is then used to compute a reconstructed vector $\hat{y}_{i}$ as:

$$
\hat{y}_{i}=f\left(W^{T} \cdot \Phi\left(x_{i}\right)+c\right)
$$

where $c$ is a $m$-dimensional bias vector. The function $f$ is an element-wise non-linear function. The hyperbolic tangent function is used as the $f$ function in the model to be able to reconstruct the vectors as close as possible to the original embeddings $X$ whose values are within the $[-1,1]$ range. Pretrained vectors are clipped to be within the the $[-1,1]$ range. This preprocess step does not alter the quality of the pretrained vectors (semantic similarity score stays the same) as most of the vector values are already within this range. 


\subsection{Objective function}

The reconstruction loss $\ell_{r e c}$ for a vector $x_{i}$ is defined as the mean squared error of $x_{i}-\hat{y}_{i}$ :

$$
\ell_{\text {rec }}\left(x_{i}\right)=\frac{1}{m} \sum_{k=0}^{m}\left(x_{i_{k}}-\hat{y}_{i_{k}}\right)^{2}
$$

where $x_{i_{k}}$ (resp. $\hat{y}_{i_{k}}$ ) represents attribute number $k$ of vector $x_{i}$ (resp. $\hat{y}_{i}$ ). The autoencoder is trained to minimize this loss for all word vectors. We noticed that this model produced good reconstructed vectors but poor semantically related binary codes as it is solely trained to minimize the reconstruction loss. The learned matrix $W$ was discarding too much similarity information from the original space in favor of the reconstruction.

This problem is solved by adding a regularization term in the objective function defined as:

$$
\ell_{\text {reg }}=\frac{1}{2}\left\|W^{T} W-I\right\|^{2}
$$

This term tends to minimize the correlations between the different features of the latent binary representation which is an essential concept in our context. Since the model aims to produce small-size binary embeddings, it needs to encode as much information as possible across all the dimensions of the binary code, therefore minimizing the correlation between the different binary features is crucial to avoid duplicate information. This regularization has the effect of preserving the information contained in the real-valued embeddings, so vectors that are close in the original space are also close in the binary space.

The balance between the influence of the reconstruction loss and the regularization loss is achieved with the $\lambda_{\text {reg }}$ parameter. The global objective function to minimize is:

$$
\mathcal{L}=\sum_{x_{i} \in X} \ell_{\text {rec }}\left(x_{i}\right)+\lambda_{\text {reg }} \ell_{\text {reg }}
$$

Note here that the same parameter matrix $W$ is both used for the encoder and for the decoder. This is actually motivated by the fact that the function $h(\cdot)$ involved in the tranformation function $\Phi(\cdot)$ is non-differentiable, so it is not possible to compute a gradient for the encoding step. It is assumed that $\frac{\partial \Phi\left(x_{i}\right)}{\partial W}=0$. However, the weights of $W$ used in the encoding step can still be updated thanks to the information provided by the decoder $\left(\frac{\partial\left(x_{i}-\hat{y}_{i}\right)^{2}}{\partial W}\right)$. The autoencoder is then trained with stochastic gradient descent (SGD) with momentum set to 0.95 . The objective function is both nonconvex and non-smooth. Despite the fact that proving a general convergence is a hard problem, we have observed in practice that the regularization plays an important role and allows our model to converge to a local optimum (without the regularization, our model oscillates and binary vectors keep changing).

\section{Experimental setup}

Several tasks have been run to measure the performance of the binary and reconstructed vectors (semantic similarity, word analogy, document classification, sentiment analysis, question classification) and another task to evaluate the computation efficiency of binary vectors (top-K queries).
Pre-trained embeddings Our autoencoder learns binary vectors from several pre-trained embeddings: dict 2 vec (Tissier, Gravier, and Habrard 2017) which contains 2.3M words and has been trained on the full English Wikipedia corpus; fasttext (Bojanowski et al. 2017) which contains $1 \mathrm{M}$ words and has also been trained on the English Wikipedia corpus; and GloVe (Pennington, Socher, and Manning 2014) which contains 400k words and has been trained on both English Wikipedia and Gigaword 5 corpora. All vectors have 300 dimensions. The learning hyperparameters used are the ones from their respective paper. Since the three embeddings are based on different methods (derivation of skip-gram for dict $2 \mathrm{vec}$ and fasttext, matrix factorization for $\mathrm{GlOVe}$ ), this demonstrates that the model is general and works for all kinds of pre-trained vectors.

Training settings Binary vectors of 4 different sizes are produced: 64, 128, 256 and 512 bits. The optimal hyperparameters are found using a grid search and selected to minimize the reconstruction loss and the regularization loss described in Section 3. The model uses a batch size of 75, 10 epochs for dict2vec and fasttext, and 5 epochs for GloVe (the autoencoder converges faster due to the smaller vocabulary) and a learning rate of 0.001 . The regularization hyperparameter $\lambda_{\text {reg }}$ depends on the starting vectors and the binary vector size. It varies from 1 to 4 in the experiments but its influence on the performance is small ( $\sim 2 \%$ variation).

Semantic word similarity Both binary and reconstructed vectors are evaluated with the standard method which consists in computing the Spearman's rank correlation coefficient between the similarity scores attributed by humans to pairs of words and the scores computed with the word vectors of these pairs. The score of real-valued vectors is the cosine similarity and the score of binary vectors is the Sokal \& Michener similarity function (Sokal 1958) defined as:

$$
\operatorname{sim}\left(v_{1}, v_{2}\right)=\frac{n_{11}+n_{00}}{n}
$$

where $n_{11}$ (resp. $n_{00}$ ) is the number of bits in $v_{1}$ and $v_{2}$ that are both set to 1 (resp. set to 0 ) simultaneously and $n$ is the vector size. The similarity datasets used are MEN (Bruni, Tran, and Baroni 2014), RW (Luong, Socher, and Manning 2013), SimLex (Hill, Reichart, and Korhonen 2015), SimVerb (Gerz et al. 2016) and WordSim (Finkelstein et al. 2001).

Word analogy This evaluation follows the standard protocol used by Mikolov et al. (2013). The task consists in finding the word $d$ in questions like " $a$ is to $b$ as $c$ is to $d$ ". The evaluation first computes the vector $v_{b}-v_{a}+v_{c}$ and then look at its closest neighbours. If the closest one is the vector associated to $d$, then the analogy is correct. The task reports the fraction of correctly guessed analogies among all analogies. For binary vectors, we replace the addition with the $\mathrm{OR}$ bitwise operator and the subtraction with the AND NOT operator because adding or subtracting bits do not really make sense in the binary space (like subtracting the bit 1 to the bit 0 ). The dataset is separated in 2: one consists of analogies about countries and currencies (semantic), the other one about english grammar (syntactic). 
Table 1: Spearman's rank correlation similarity and word analogy scores for binary vectors (bin) of 64, 128, 256 and 512 bits, reconstructed real-valued vectors from the binary codes ( $(\mathrm{eec})$ and binary vectors produced with Local Sensitive Hashing (LSH). For each dataset, scores of original real-valued vectors are also reported ( raw column).

\begin{tabular}{|c|c|c|c|c|c|c|c|c|c|c|c|c|c|c|c|}
\hline & \multicolumn{5}{|c|}{ dict2vec } & \multicolumn{5}{|c|}{ fasttext } & \multicolumn{5}{|c|}{ GloVe } \\
\hline & raw & 64 & 128 & 256 & 512 & raw & 64 & 128 & 256 & 512 & raw & 64 & 128 & 256 & 512 \\
\hline MEN & 74.6 & & & & & 80.7 & & & & & 73.7 & & & & \\
\hline bin & - & 66.1 & 71.3 & 70.3 & 71.3 & - & 57.9 & 72.0 & 75.9 & 76.3 & - & 46.1 & 63.3 & 69.4 & 72.7 \\
\hline rec & - & 64.5 & $\underline{69.6}$ & 67.8 & 64.7 & - & 45.8 & 56.2 & $\underline{62.3}$ & 59.3 & - & 43.6 & 50.5 & 68.5 & 72.2 \\
\hline LSH & - & 47.7 & $\overline{56.2}$ & 62.6 & 67.8 & - & 47.7 & 60.7 & $\overline{70.0}$ & 75.0 & - & 50.8 & 62.0 & 64.9 & $\overline{71.0}$ \\
\hline RW & 50.5 & & & & & 53.8 & & & & & 41.2 & & & & \\
\hline bin & - & 36.5 & 42.0 & 45.6 & 45.6 & - & 36.8 & 44.7 & 52.7 & 52.7 & - & 25.1 & 34.3 & 40.7 & 40.2 \\
\hline rec & - & 35.7 & 41.6 & $\underline{44.6}$ & 39.3 & - & 28.9 & 31.6 & 45.7 & 44.1 & - & 24.8 & 29.2 & 36.4 & 40.5 \\
\hline LSH & - & 26.4 & 37.2 & $\overline{41.7}$ & 46.2 & - & 34.5 & 40.3 & $\overline{47.5}$ & 46.5 & - & 26.3 & 33.0 & 35.8 & $\overline{38.3}$ \\
\hline SimLex & 45.2 & & & & & 44.1 & & & & & 37.1 & & & & \\
\hline bin & - & 32.0 & 38.1 & 44.8 & 42.9 & - & 25.1 & 38.0 & 44.6 & 43.0 & - & 20.5 & 31.4 & 37.2 & 36.8 \\
\hline rec & - & 30.4 & 37.9 & $\underline{42.4}$ & 39.3 & - & 19.2 & 30.0 & $\underline{40.5}$ & 34.0 & - & 19.6 & 19.1 & 34.2 & $\underline{38.2}$ \\
\hline LSH & - & 29.6 & 35.9 & 40.2 & 39.5 & - & 28.7 & 32.0 & 38.6 & 41.1 & - & 24.7 & 30.5 & 33.1 & 34.6 \\
\hline SimVerb & 41.7 & & & & & 35.6 & & & & & 22.7 & & & & \\
\hline bin & - & 25.3 & 36.6 & 38.4 & 35.5 & - & 19.2 & 26.7 & 33.7 & 35.1 & - & 7.8 & 18.7 & 22.9 & 23.0 \\
\hline rec & - & 23.7 & 35.4 & $\underline{37.5}$ & 29.4 & - & 12.8 & 18.2 & $\underline{25.6}$ & 25.3 & - & 8.0 & 12.4 & 22.1 & 24.7 \\
\hline LSH & - & 22.0 & 27.5 & $\overline{31.6}$ & 36.9 & - & 20.5 & 23.3 & $\overline{30.7}$ & 30.2 & - & 14.6 & 17.4 & 18.8 & $\overline{20.7}$ \\
\hline WS353 & 72.5 & & & & & 69.7 & & & & & 60.9 & & & & \\
\hline bin & - & 63.7 & 71.6 & 69.6 & 66.6 & - & 50.3 & 69.1 & 70.0 & 70.3 & - & 30.1 & 44.9 & 56.6 & 60.3 \\
\hline rec & - & 61.4 & $\underline{69.0}$ & 67.4 & 58.8 & - & 36.5 & 53.6 & $\underline{64.0}$ & 53.6 & - & 26.5 & 42.2 & 56.5 & 62.0 \\
\hline LSH & - & 45.5 & $\overline{56.9}$ & 64.9 & 65.5 & - & 46.7 & 53.2 & $\overline{58.6}$ & 63.8 & - & 41.1 & 44.4 & 50.5 & $\overline{57.8}$ \\
\hline Sem. analogy & 59.6 & & & & & 37.6 & & & & & 77.4 & & & & \\
\hline bin & - & 2.6 & 12.0 & 26.7 & 30.1 & - & 2.3 & 7.5 & 18.0 & 25.0 & - & 8.5 & 26.7 & 53.4 & 65.3 \\
\hline rec & - & 2.6 & 10.2 & 22.8 & 30.9 & - & 1.8 & 5.0 & 14.6 & 15.2 & - & 7.7 & 23.0 & 49.1 & 62.8 \\
\hline LSH & - & 0.8 & 4.6 & 14.9 & $\overline{29.9}$ & - & 0.8 & 6.4 & 13.0 & $\overline{20.4}$ & - & 6.1 & 25.9 & 47.9 & $\overline{59.3}$ \\
\hline Syn. analogy & 54.0 & & & & & 87.4 & & & & & 67.0 & & & & \\
\hline bin & - & 3.5 & 16.7 & 34.8 & 36.2 & - & 8.0 & 34.5 & 57.3 & 64.7 & - & 7.3 & 23.9 & 46.3 & 52.4 \\
\hline rec & - & 3.6 & 16.1 & 31.2 & $\underline{37.5}$ & - & 4.6 & 14.6 & 50.8 & $\underline{53.1}$ & - & 7.3 & 21.7 & 44.6 & $\underline{54.0}$ \\
\hline LSH & - & 1.7 & 7.8 & 23.4 & $\overline{35.8}$ & - & 4.0 & 21.5 & 46.2 & 65.7 & - & 5.6 & 21.6 & 39.1 & 52.3 \\
\hline
\end{tabular}

Document/Question classification and sentiment analysis The evaluation follows the same protocol as described in the literature (Zhang, Zhao, and LeCun 2015; Joulin et al. 2017) which consists in predicting the assigned label given the bag-of-words representation of a text. A single hidden layer neural network where the input weights have been initialized with the binary or reconstructed vectors is used. The input weights are fixed during training so that the classification accuracy only depends on the vectors used to initialize the neural network. The datasets used are AG-News and DBpedia for document classication, Yahoo Answers for question classification and Amazon and Yelp reviews (both polarity and full) for the sentiment analysis task. Each dataset is split into a training and a test file and the same training and test files are used for all word embedding models. Accuracy results are reported in Table 2.

Top-K queries performances A top-K query consists in finding the $K$ closest vectors given a single word vector query. The closest vectors are the ones with the highest similarity with the query vector (Sokal \& Michener similarity for binary vectors, cosine similarity for real-valued ones). The top- $\mathrm{K}$ vectors are found by performing a linear scan across all vectors. Two execution times are measured for both binary and real-valued vectors: the time it takes to get the results once the vectors are loaded in memory and the time it takes to load the vectors and perform the query.

\section{Results and binary vectors analysis 5.1 Binary embeddings performances}

Semantic word similarity Table 1 reports the Spearman's rank correlation scores obtained with the binarized vectors (bin) and the scores of the original real-valued vectors (raw) whose size is 9600 bits (300 dimensions, 32-bit floating-point values). The best scores for binarized vectors are reached with 256 or 512 bits. For fasttext and GloVe, the results are very close to the scores obtained with the raw vectors (absolute difference smaller than 1 point). For dict $2 \mathrm{vec}$, the deviation is larger (between 3 and 5 points) but still in the same order of magnitude.

Binarizing word vectors can lead to better scores compared to the original real-valued ones (the raw column). For instance, the 512-bit version of fasttext on WS353 (70.3 against 69.7) or the 256-bit version of GloVe on SimVerb (22.9 against 22.7). Moreover, the 64-bit version of dict2vect is better than the real-valued GloVe embedding (which uses 9600 bits of information per vector, so the binary codes are 150 times smaller) on SimVerb (25.3 against 22.7) and WS353 (63.7 against 60.9). This demonstrates that the method can produce rich semantically related small binary codes but more generally, that binary vectors can have the same semantic information as the real-valued vectors.

The average semantic correlation scores (aggregated with the Fisher's transformation) of binary and original vectors 
Table 2: Document classification (top), question classification (middle) and sentiment analysis (bottom) accuracies for binary vectors (bin) of 64, 128, 256 and 512 bits, reconstructed real-valued vectors (rec) and binary vectors produced with Local Sensitive Hashing (LSH). For each dataset, scores of original real-valued vectors are also reported (raw column).

\begin{tabular}{|c|c|c|c|c|c|c|c|c|c|c|c|c|c|c|c|}
\hline & \multicolumn{5}{|c|}{ dict2vec } & \multicolumn{5}{|c|}{ fasttext } & \multicolumn{5}{|c|}{ GloVe } \\
\hline & raw & 64 & 128 & 256 & 512 & raw & 64 & 128 & 256 & 512 & raw & 64 & 128 & 256 & 512 \\
\hline AG-News & 89.0 & & & & & 86.9 & & & & & 89.5 & & & & \\
\hline bin & - & 85.3 & 85.9 & 87.7 & 87.8 & - & 84.5 & 85.9 & 87.3 & 87.7 & - & 84.0 & 87.2 & 88.5 & 88.5 \\
\hline rec & - & 85.2 & 86.3 & $\underline{87.9}$ & 87.2 & - & 82.8 & 84.3 & $\underline{87.7}$ & 87.3 & - & 83.9 & 87.7 & 88.6 & $\underline{89.2}$ \\
\hline LSH & - & 78.8 & 82.6 & $\overline{86.1}$ & 88.1 & - & 77.5 & 83.3 & $\overline{86.1}$ & 88.8 & - & 83.5 & 86.6 & 88.4 & $\overline{88.6}$ \\
\hline DBpedia & 97.6 & & & & & 95.0 & & & & & 97.2 & & & & \\
\hline bin & - & 94.1 & 96.1 & 97.0 & 97.3 & - & 91.7 & 95.1 & 96.6 & 97.3 & - & 90.9 & 95.0 & 96.8 & 97.2 \\
\hline$r e c$ & - & 94.0 & 95.9 & 96.8 & 96.6 & - & 89.5 & 92.6 & $\underline{96.4}$ & 96 & . & 91.2 & 95.2 & 96.8 & 97.0 \\
\hline LSH & - & 89.6 & 94.2 & $\overline{96.5}$ & 97.4 & - & 87.4 & 93.8 & $\overline{96.2}$ & 97.2 & 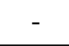 & 90.4 & 94.2 & 96.3 & $\overline{97.2}$ \\
\hline Yahoo Ans & 68.1 & & & & & 67.2 & & & & & 68.1 & & & & \\
\hline bin & - & 60.7 & 63.8 & 66.0 & 66.8 & - & 60.4 & 63.9 & 66.4 & 67.8 & - & 57.5 & 62.5 & 66.4 & 66.1 \\
\hline rec & - & 60.8 & 63.8 & 65.9 & 66.0 & 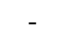 & 60.0 & 62.9 & 66.3 & 66.8 & . & 58.4 & 64.3 & 66.7 & 67.0 \\
\hline LSH & - & 52.3 & 59.9 & 64.5 & $\overline{67.1}$ & - & 52.2 & 59.5 & 64.9 & $\overline{66.9}$ & - & 56.8 & 62.0 & 65.3 & $\overline{67.0}$ \\
\hline mazon Full & 47.5 & & & & & 49.0 & & & & & 47.1 & & & & \\
\hline bin & - & 39.9 & 43.9 & 46.8 & 47.7 & - & 39.0 & 43.9 & 47.9 & 49.8 & - & 37.4 & 42.6 & 46.7 & 47.8 \\
\hline rec & - & 40.1 & 44.0 & 46.8 & 46.2 & - & 39.1 & 43.8 & 48.1 & 48.5 & - & 39.8 & 45.3 & 47.1 & $\underline{47.3}$ \\
\hline LSH & - & 38.3 & 42.5 & $\overline{45.6}$ & 48.1 & - & 38.6 & 42.7 & 47.3 & $\overline{49.5}$ & - & 37.9 & 43.0 & 45.9 & $\overline{48.6}$ \\
\hline Amazon Pol & 84.2 & & & & & 85.6 & & & & & 83.8 & & & & \\
\hline bin & - & 76.3 & 80.7 & 83.2 & 83.8 & - & 75.1 & 80.2 & 84.5 & 85.8 & - & 73.1 & 78.9 & 83.2 & 84.4 \\
\hline rec & - & 76.6 & 80.8 & 83.2 & 82.4 & - & 75.1 & 80.2 & 84.7 & 84.8 & - & 76.6 & 80.2 & 83.6 & 83.7 \\
\hline LSH & - & 74.3 & 79.0 & $\overline{81.9}$ & 84.5 & - & 73.8 & 78.5 & 83.4 & $\overline{85.7}$ & - & 74.7 & 79.1 & 82.1 & $\overline{85.0}$ \\
\hline Yelp Full & 52.5 & & & & & 52.1 & & & & & 52.7 & & & & \\
\hline bin & - & 45.1 & 48.7 & 51.6 & 52.0 & - & 44.2 & 49.7 & 53.0 & 54.6 & - & 42.7 & 48.4 & 51.8 & 53.2 \\
\hline rec & - & 45.3 & 48.8 & $\underline{51.6}$ & 50.9 & - & 43.5 & 47.8 & 53.0 & 53.1 & - & 43.4 & 50.3 & 52.3 & $\underline{52.8}$ \\
\hline LSH & - & 43.0 & 47.7 & $\overline{51.0}$ & 53.1 & - & 44.3 & 47.6 & 52.4 & $\overline{54.3}$ & - & 43.6 & 48.2 & 51.5 & $\overline{53.4}$ \\
\hline Yelp Pol & 87.8 & & & & & 88.0 & & & & & 87.9 & & & & \\
\hline bin & - & 80.8 & 84.5 & 86.6 & 87.6 & - & 80.1 & 84.5 & 88.1 & 89.5 & - & 77.8 & 84.2 & 86.9 & 88.7 \\
\hline rec & - & 80.9 & 84.5 & 86.6 & 86.1 & - & 79.6 & 84.0 & 88.2 & 88.5 & - & 78.6 & 85.7 & 87.5 & 87.7 \\
\hline LSH & - & 77.9 & 82.8 & $\overline{86.1}$ & 88.0 & - & 80.3 & 82.2 & 87.2 & $\overline{89.8}$ & - & 79.0 & 83.1 & 86.6 & $\overline{88.6}$ \\
\hline
\end{tabular}

(whose size is 9600 bits per vector) are plotted in Figure 2. The 512-bit version of GloVe is on par with the real-valued version. However the performance loss is greater for fasttext and dict $2 \mathrm{vec}$ vectors. These embeddings contain $1 \mathrm{M}$ of words for fasttext, and $2.3 \mathrm{M}$ for dict2vec, whereas GloVe only contains $400 \mathrm{k}$ words. As the binary space has a finite size depending on the binary vector size, it becomes harder for the autoencoder to find a distribution of the binary vectors that preserves the semantic similarity when the number of vectors increases.

Word Analogy Table 1 reports the word analogy accuracies of binary vectors for both semantic and syntactic analogies. Although the best scores are also obtained with larger binary codes (512 bits), the scores are lower than the realvalued vectors. This can be explained by the fact that the task of word analogy is not suited to evaluate binary vectors. With real-valued vectors, the analogies are found with vector addition and subtraction in the $\mathcal{R}^{d}$ space. In the binary space (where each value is either 0 or 1 ), adding or subtracting vectors does not make sense (like subtracting the bit 1 from the bit 0 ) resulting in poor accuracies for the word analogies with the binary vectors.

Text classification The accuracies obtained on the different datasets of the document classification task for both binarized vectors (bin) and original vectors ( raw) are provided in Table 2. For this task, the binarized vectors achieve the best scores with 512 bits in general. Similarly to the semantic similarity task, the binary vectors are sometimes better than the original vectors. This is especially true for the fasttext binarized vectors where the accuracies goes from 95.0 to 97.3 on DBpedia, or goes from 49.0 to 49.8 on Amazon Full reviews.

Smaller sizes of binary vectors lead to better compression rates but cause a slight decrease in performance. The 256bit vectors of GloVe are 37.5 times smaller than the original vectors (whose size is 9600 bits) but have an accuracy drop between $0.4 \%$ and $2.5 \%$ depending on the dataset. The 64-bit vectors of dict2vec (compression rate of 150) have a loss of accuracy of about $4 \%$ on AG-News and DBpedia, about $11 \%$ on Yahoo answers and about $16 \%$ on Amazon Full reviews. The two latter datasets are the bigger ones with $3 \mathrm{M}$ training samples for Amazon Full reviews and 1.4M for Yahoo answers while AG-News and DBpedia respectively contain $120 \mathrm{k}$ and $560 \mathrm{k}$ training samples. As the dataset becomes larger, the information required to correctly classify the documents also increases, so is the accuracy loss on these datasets when small binary vectors are used. Overall, this experiments show once again that our binary embeddings are competitive in comparison with real-valued ones. 

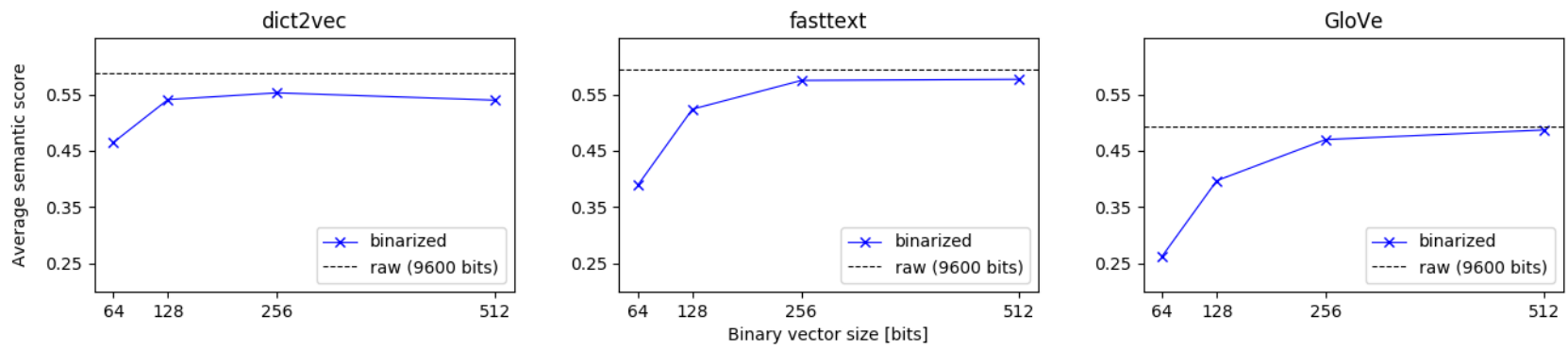

Figure 2: Fisher's transformed average semantic correlation scores for different binary vector size for all type of embeddings. The raw baseline indicates the score obtained with real-valued embeddings.

\subsection{Reconstructed vectors performances}

Table 1 and Table 2 also respectively report the scores obtained on semantic word similarity and analogy and text classification tasks using the reconstructed $(r e c)$ vectors.

On the semantic similarity task, fasttext and dict2vec mostly best operate using 256-bit binary vectors while GloVe requires larger binary vectors (512-bit) to reconstruct good real-valued vectors. For most datasets, the reconstructed vectors from the binarized GloVe 512-bit representations outperforms the original real-valued vectors: 38.2 against 37.1 on SimLex, 24.7 against 22.7 on SimVerb and 62.0 against 60.9 on WS353. For dict 2 vec and fasttext, binarizing and then reconstructing real-valued vectors from the binary ones causes a loss of performance compared to the original word vectors: between $-4.8 \%$ (WS353) and $11.7 \%$ (RW) for dict2vec; between $-8.2 \%$ (WS353) and $28.1 \%$ (SimVerb) for fasttext. The performance loss of reconstructed vectors is larger for fasttext than for dict2vec due to their different embedding scheme. Fasttext also encodes additional morphological information in the word vector space by considering that a word is the sum of its subwords, which is harder to reconstruct after a dimension reduction (the binarization).

On NLP application tasks like document classification or sentiment analysis (Table 2), the results are very consistent: the reconstructed vectors exhibit close performances to the binary representations, which in turn exhibit (almost) equal performances to the original vectors - whatever the initial embedding model. Although the pairwise word semantic similarity is not perfectly preserved in reconstructed vectors, the document classification task only needs vectors close enough for those of the same category but not necessarily very accurate within a category. This makes the binary or reconstructed vectors good for a real use case NLP task. The optimal number of bits required to recontruct good realvalued vector is the same as for the semantic similarity task (256 bits for dict2vec/fasttext, 512 for GloVe).

\subsection{Speed improvements in top-K queries}

The execution time (in milliseconds) for some top-K queries benchmarks on GloVe vectors are reported in Table 3. The first three rows (Top 1, Top 10, Top 50) indicate the time used by the program to perform the query after the vectors are loaded i.e. the time to linearly scan all the vectors, compute the similarity with the query vector and select the $\mathrm{K}$ highest values. Finding the closest 10 words with 256-bit vectors is 30 times faster compared to using real-valued vectors and can be up to 36 times faster with the 64-bit vectors.

Having faster computations is not the only interest of binary vectors. Since they take less space in memory, they are also much faster to load. The last line in Table 3 indicates the time needed to load the vectors from a file and perform a top-10 query. It takes 310 milliseconds to load the 256-bit binary vectors and run the query whereas it take $23.5 \mathrm{sec}-$ onds for the real-valued ones, which is 75 times slower.

Table 3: Execution time (in $\mathrm{ms}$ ) to run a top-K query on binary and real-valued vectors.

\begin{tabular}{cccccc}
\hline Execution time $(\mathrm{ms})$ & 64-bit & 128-bit & 256-bit & 512-bit & Real-valued \\
\hline Top 1 & 2.71 & 2.87 & 3.23 & 4.28 & 97.89 \\
Top 10 & 2.72 & 2.89 & 3.25 & 4.29 & 98.08 \\
Top 50 & 2.75 & 2.91 & 3.27 & 4.32 & 98.44 \\
Loading + Top 10 & 160 & 213 & 310 & 500 & 23500 \\
\hline
\end{tabular}

\subsection{Comparison with the naive approach}

A naive approach to produce binary vectors is to map negative values of pre-trained word embeddings to 0 and positive values to 1 . Unfortunately the binary vectors produced with this method have the same number of dimensions as the original vectors and since most pre-trained vectors are not available in 64,128 or 256 dimensions, the binary vectors are not aligned with CPU registers (see Section 1) which is an important requirement to achieve fast vector operations.

To produce aligned vectors with the naive method, one has first to train real-valued embeddings with 64, 128 or 256 dimensions and then apply a naive binarization function to all vector values. First, this process is slower than our method. It requires 8 hours while we only need 13 minutes. Second, binary vectors produced with the naive binarization perform worse compared to those produced with the proposed method. The Fisher's average semantic score is 44.7 and 49.6 for the naive 64 and 128 bits vectors while the 64 and 128 bits dict2vec vectors achieve 46.5 and 54.1. 


\subsection{Comparison with other binarization methods}

We used the Locality Sensitive Hashing method to produce binary vectors of $64,128,256$ and 512 bits from the same real-valued vectors as us and have evaluated them on the same tasks (Table 1 and 2). For the word similarity and analogy tasks, our vectors are always better (e.g. 70.3 vs. 63.8 for fasttext 512-bit on WS353, 34.8 vs. 23.4 for dict2vec 256-bit on Syn. analogy) except for GloVe 64-bit and some fasttext 64-bit vectors. For the classification task, our 512-bit vectors are on par with the LSH ones, but our 128 and 256 bits vectors have better performances than the respective LSH ones. Our model is better suited when the compression is higher and gives the best size to performance ratio.

We also used the Faruqui method (2015) to produce binary vectors. Faruqui's vectors are mostly sparse $(90 \%$ of bits set to 0 ) but not very small to be computationally interesting: dimension is set to 3000-bit, so they do not fit in registers. They are also have poorer performance than our binary vectors: 0.560 for their average score on semantic similarity while we achieve 0.575 with our 256 -bit vectors.

\subsection{Qualitative analysis of binary embeddings}

Evolution of related word vectors similarity The semantic word similarity task rely on specific datasets. They contain pairs of words and a value assigned by humans (e.g. computer/keyboard - 0.762)). This value represents the semantic similarity between the two words and is assumed to be the ground truth that word embeddings should encode. The cosine similarity of the pre-trained vectors of the words of one pair does not always match the value associated to this pair, but binarizing the vectors helps to move closer the vector similarity to the value, making the embedding closer to the human judgment. Some pairs are reported in Table 4.

Table 4: Semantic similarity for some pairs of words, evaluated by human or computed with binary/real-valued vectors.

\begin{tabular}{cccc}
\hline Words & Human judgment & Binary & Cosine \\
\hline dollar - buck & 0.92 & 0.56 & 0.13 \\
seafood - sea & 0.75 & 0.62 & 0.24 \\
money - salary & 0.79 & 0.58 & 0.41 \\
car - automobile & 0.98 & 0.69 & 0.60 \\
\hline
\end{tabular}

Visualization of activated dimensions in binary vectors In Figure 3, the 50 most similar (top) and the 50 least similar (bottom) 512-bit binary vectors of some words are plotted. Each pixel is either red or white depending on whether it is 1 or 0 . Some vertical stripes are clearly visible in the top area: similar word vectors have the same bits enabled for certain dimensions and therefore share the same color of pixel for those dimensions. This is especially true for the closest words of "automobile". In the bottom area, no vertical patterns are visible because two non-similar binary vectors do not have the same enabled bits. This visualization shows that the binarized vectors have semantic properties similar to real-valued embeddings.
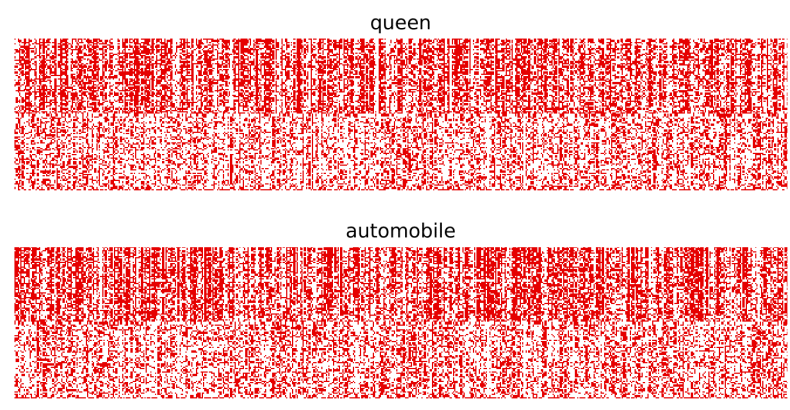

jupiter

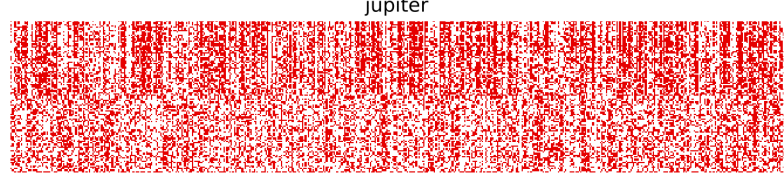

man

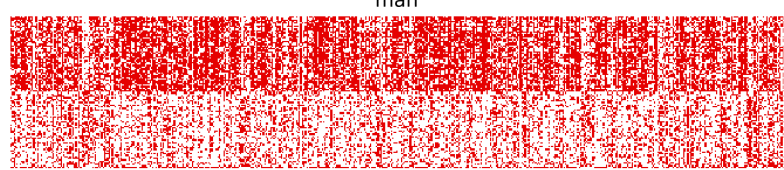

Figure 3: Representation of the activated bits in some binary vectors for the words queen, automobile, jupiter and man. For each word, its 50 closest neighbors (top) and its 50 furthest neighbors (bottom) are plotted. Red pixels represent the bits set to 1 and white pixels the bits set to 0 .

\section{Conclusion}

This article presents a new encoder/decoder architecture for transforming real-valued vectors into binary vectors of any size. This is particularly suitable when the size of the binary vectors corresponds to the CPU cache sizes as it allows a significant increase in vector operation computing speed. Our method has the advantage of being simple yet powerful and allows to keep semantic information in binary vectors.

Our binary embeddings exhibit almost the same performances as the original real-valued vectors on both semantic similarity and document classification tasks. Furthermore, since the binary representations are smaller (a 256-bit binary code is 37.5 smaller than a traditional 300-dimensional word vector), it allows one to run a top-K query 30 times faster than with real-valued vectors since the binary distance is much faster to compute than a regular cosine distance. Additionally, it is possible to recontruct real-valued vectors from the binary representations using the model decoder.

\section{References}

Bengio, Y.; Ducharme, R.; Vincent, P.; and Jauvin, C. 2003. A neural probabilistic language model. Journal of machine learning research 3(Feb):1137-1155.

Bojanowski, P.; Grave, E.; Joulin, A.; and Mikolov, T. 2017. Enriching word vectors with subword information. Transactions of the Association of Computational Linguistics 5:135146. 
Bruni, E.; Tran, N.-K.; and Baroni, M. 2014. Multimodal distributional semantics. J. Artif. Intell. Res.(JAIR) 49(1-47).

Charikar, M. S. 2002. Similarity estimation techniques from rounding algorithms. In Proceedings of the thiry-fourth annual ACM symposium on Theory of computing, 380-388. ACM.

Chen, T.; Min, M. R.; and Sun, Y. 2018. Learning k-way d-dimensional discrete codes for compact embedding representations. In Dy, J., and Krause, A., eds., Proceedings of the 35th International Conference on Machine Learning, volume 80 of Proceedings of Machine Learning Research, 854-863. Stockholmsmässan, Stockholm Sweden: PMLR.

Conneau, A.; Schwenk, H.; Barrault, L.; and Lecun, Y. 2017. Very deep convolutional networks for text classification. In Proceedings of the 15th Conference of the European Chapter of the Association for Computational Linguistics: Volume 1, Long Papers, 1107-1116. Valencia, Spain: Association for Computational Linguistics.

Faruqui, M.; Tsvetkov, Y.; Yogatama, D.; Dyer, C.; and Smith, N. 2015. Sparse overcomplete word vector representations. In Proceedings of the 53rd Annual Meeting of the Association for Computational Linguistics and the 7th International Joint Conference on Natural Language Processing (Volume 1: Long Papers), 1491-1500. Beijing, China: Association for Computational Linguistics.

Finkelstein, L.; Gabrilovich, E.; Matias, Y.; Rivlin, E.; Solan, Z.; Wolfman, G.; and Ruppin, E. 2001. Placing search in context: The concept revisited. In Proceedings of the 10th international conference on World Wide Web, 406414. ACM.

Gerz, D.; Vulić, I.; Hill, F.; Reichart, R.; and Korhonen, A. 2016. Simverb-3500: A large-scale evaluation set of verb similarity. In Proceedings of the 2016 Conference on Empirical Methods in Natural Language Processing, 2173-2182. Austin, Texas: Association for Computational Linguistics.

Hill, F.; Reichart, R.; and Korhonen, A. 2015. Simlex-999: Evaluating semantic models with (genuine) similarity estimation. Computational Linguistics 41(4):665-695.

Hubara, I.; Courbariaux, M.; Soudry, D.; El-Yaniv, R.; and Bengio, Y. 2016. Binarized neural networks. In Advances in neural information processing systems, 4107-4115.

Joulin, A.; Grave, E.; Bojanowski, P.; Douze, M.; Jégou, H.; and Mikolov, T. 2016. Fasttext. zip: Compressing text classification models. arXiv preprint arXiv:1612.03651.

Joulin, A.; Grave, E.; Bojanowski, P.; and Mikolov, T. 2017. Bag of tricks for efficient text classification. In Proceedings of the 15th Conference of the European Chapter of the Association for Computational Linguistics: Volume 2, Short Papers, 427-431. Valencia, Spain: Association for Computational Linguistics.

Ling, S.; Song, Y.; and Roth, D. 2016. Word embeddings with limited memory. In Association of Computational Linguistics.

Luong, M.-T.; Socher, R.; and Manning, C. D. 2013. Better word representations with recursive neural networks for morphology. In CoNLL, 104-113.
Ma, X., and Hovy, E. 2016. End-to-end sequence labeling via bi-directional lstm-cnns-crf. In Proceedings of the 54th Annual Meeting of the Association for Computational Linguistics (Volume 1: Long Papers), 1064-1074. Association for Computational Linguistics.

Mikolov, T.; Sutskever, I.; Chen, K.; Corrado, G. S.; and Dean, J. 2013. Distributed representations of words and phrases and their compositionality. In Advances in neural information processing systems, 3111-3119.

Norouzi, M.; Punjani, A.; and Fleet, D. J. 2012. Fast search in hamming space with multi-index hashing. In Computer Vision and Pattern Recognition (CVPR), 2012 IEEE Conference on, 3108-3115. IEEE.

Pennington, J.; Socher, R.; and Manning, C. 2014. Glove: Global vectors for word representation. In Proceedings of the 2014 conference on empirical methods in natural language processing (EMNLP), 1532-1543.

Qian, Q.; Huang, M.; Lei, J.; and Zhu, X. 2017. Linguistically regularized $1 \mathrm{stm}$ for sentiment classification. In Proceedings of the 55th Annual Meeting of the Association for Computational Linguistics (Volume 1: Long Papers), 16791689. Vancouver, Canada: Association for Computational Linguistics.

Raunak, V. 2017. Effective dimensionality reduction for word embeddings. NIPS 2017, workshop on Learning with Limited Labeled Data.

Shen, D.; Su, Q.; Chapfuwa, P.; Wang, W.; Wang, G.; Henao, R.; and Carin, L. 2018. Nash: Toward end-to-end neural architecture for generative semantic hashing. In Proceedings of the 56th Annual Meeting of the Association for Computational Linguistics (Volume 1: Long Papers), 2041-2050. Association for Computational Linguistics.

Shu, R., and Nakayama, H. 2018. Compressing word embeddings via deep compositional code learning. In International Conference on Learning Representations.

Socher, R.; Perelygin, A.; Wu, J.; Chuang, J.; Manning, C. D.; Ng, A.; and Potts, C. 2013. Recursive deep models for semantic compositionality over a sentiment treebank. In Proceedings of the 2013 conference on empirical methods in natural language processing, 1631-1642.

Sokal, R. R. 1958. A statistical method for evaluating systematic relationship. University of Kansas science bulletin 28:1409-1438.

Subercaze, J.; Gravier, C.; and Laforest, F. 2015. On metric embedding for boosting semantic similarity computations. In Association of Computational Linguistics.

Tissier, J.; Gravier, C.; and Habrard, A. 2017. Dict2vec: Learning word embeddings using lexical dictionaries. In Conference on Empirical Methods in Natural Language Processing (EMNLP 2017), 254-263.

Xu, J.; Wang, P.; Tian, G.; Xu, B.; Zhao, J.; Wang, F.; and Hao, H. 2015. Convolutional neural networks for text hashing. In IJCAI, 1369-1375.

Zhang, X.; Zhao, J.; and LeCun, Y. 2015. Character-level convolutional networks for text classification. In Advances in neural information processing systems, 649-657. 